\title{
El terapeuta no nace, se hace.
}

Therapists are not born but made.

\author{
Daniel M. Campagne a. \\ ${ }^{a}$ UNED, Facultad de Psicología, Departamento de Personalidad, Evaluación y Tratamiento Psicológicos, \\ Madrid, España.
}

Correspondencia: Daniel M. Campagne (dmcampagne@gmail.com)

Recibido: 26/02/2013; aceptado: 22/06/2013

\begin{abstract}
RESUMEN: A finales del siglo pasado se había acumulado evidencia suficiente para la identificación científica de los componentes comunes del género terapia psicológica y asignarles un peso relativo medido y verificado. El resultado subraya que el éxito de la terapia depende no del terapeuta sino del cliente. Con ello, se consolidó el fundamento de una psicoterapia moderna que, aún respetando las diversas escuelas y técnicas, ahora se guía más por hechos contrastados que por supuestos teóricos, por muy convincentes que éstos suenen. Sin embargo, no son sólo los datos que pueden ser contrastados los que deben guiar al profesional, y la investigación cualitativa se abre camino para completar datos cuantitativos no tan claros. Los cuatro factores comunes que indican lo que realmente funciona en psicoterapia son de obligatorio conocimiento para los que se dedican a la terapia, tanto clínica como educativa o de crecimiento personal.

PALABRAS CLAVE: Psicoterapia; eficacia; factores comunes; cliente; crecimiento personal.
\end{abstract}

ABSTRACT: At the end of the last century, sufficient evidence had been collected to scientifically identify the common factors of psychological therapy in general, and provide them with a relative measured and contrasted weight. The results indicate that therapy efficacy depends not on the therapist but on the client. This gave a solid base to modern psychotherapy which, with due respect to different schools and techniques, now is guided more by validated facts than by theoretical suppositions, however convincing these seem. The four common factors of what really works in psychotherapy are necessary knowledge for therapy professionals, both clinical and educational or in personal growth.

KEY WORDS: Psychotherapy; efficacy; common factors; client; personal growth.

\section{Introducción}

La identificación y el perfeccionamiento de habilidades, procesos y actitudes que hacen que funcione la psicoterapia, independientemente de la clase de terapia o del modelo terapéutico, ha sido un trabajo arduo y prolongado en el tiempo y que no ha terminado. En este artículo se refleja a grandes rasgos la historia de este esfuerzo compartido y se recopila parte de las recomendaciones o, mejor dicho, las bases necesarias para lo que actualmente puede considerarse una actitud competente del psicoterapeuta. Algunas de estas "bases necesarias" o "verdades profesionales" puede que no sean del agrado del terapeuta que se considera el principal actor en su trabajo. Verse confrontado con las elocuentes conclusiones de tantos estudios $\mathrm{y}$ tener que reconocer que son los factores del cliente y no las artes del terapeuta los que determinan la eficacia de la terapia, requiere realismo y humildad. 
ORIGINALES Y REVISIONES

\section{La identificación de lo que sirve en terapia}

La American Psychological Association (APA) publicó en 1999 el libro The heart and soul of change: What works in therapy (Corazón y alma del cambio: Lo que funciona en terapia), de los prestigiosos autores Mark Hubble, Barry Duncan \& Scott Miller (1), siendo una compilación de datos y referencias que es fuente obligada para el estudio de la eficacia terapéutica en todos sus aspectos y cuyas conclusiones han sido apoyadas por posteriores publicaciones de primer orden (2). En 2012, la APA publicó la Resolución sobre el Reconocimiento de la Eficacia de la Psicoterapia la cual refleja el estado actual de lo que se sabe, con una lista de las investigaciones de la última década (3). La presente revisión recoge el argumento central de estas publicaciones sobre la eficacia demostrada de la terapia, y del rol del terapeuta en el proceso terapéutico.

Saul Rozenzweig, "padre" de la identificación de los factores comunes, y compañero de la Universidad de Harvard del conductista B.F.Skinner decía, ya en 1936, que "La eficacia de las diferentes aproximaciones terapéuticas tiene más relación con sus elementos comunes que con sus bases teóricas" (4). Jung, unos años después, afirmó lo contrario: "No es la terapia, sino el terapeuta". El convencimiento del influyente Jung, ahora y desde hace decenios demostrado erróneo, creó varias generaciones de psicoterapeutas que se creían - y algunos siguen creyendo - magos de la mente humana.

La psicoterapia empezó su historia moderna centrándose en el funcionamiento interpersonal, tal como lo reflejan las teorías psicodinámicas de Viena. A mediados del siglo veinte la psicología conductista estadounidense mostraba el afán de tecnificar el tema y así limitar la experiencia humana a unas categorías reducidas y limitadas por unas pocas leyes "universales" sobre la conducta. La mayoría de la evidencia empírica ahora disponible indica que haríamos bien volviendo a la comprensión de nuestra intersubjetividad esencial, de la importancia fundamental de las relaciones, y del potencial curador de una alianza terapéutica bien llevada. Sin embargo, a la vez se aprecia una re-impulsada tendencia a considerar modelos mecánicos y reduccionistas de la mente, bajo el lema de responsabilidad y eficacia clínica, y motivado más por motivos económicos y políticos que por evidencia científica (1:165).

Hans Eysenck, en los años 50 y 60 del pasado siglo, dinamitó la autoconfianza de los terapeutas y de los modelos competidores de terapia de entonces, con un análisis de los resultados de diversas terapias y afirmó que, aunque aproximadamente dos terceras partes de los clientes con un diagnóstico de neurosis mejoró sustancialmente dentro de los primeros dos años de terapia, una proporción parecida mejoró, en un período comparable, sin ninguna terapia (5-6).

No era la única voz crítica con el modelo terapéutico entonces vigente. Jerome Frank, catedrático de la universidad Johns Hopkins y asiduo comparatista en 
ORIGINALES Y REVISIONES

temas de la eficacia de las diversas aproximaciones terapéuticas, observó en 1973 que "La psicoterapia es el único tratamiento que crea la enfermedad que pretende curar" (7). En un tiempo relativamente corto, la psicoterapia se hacía con un mercado importante, así como con un fundamento teórico diversificado. Los números así lo demuestran: las categorías del DSM (Manual diagnóstico y estadístico de las enfermedades mentales - American Psychiatric Association) subieron de los 66 trastornos mentales que catalogaba la primera edición a 286 en la cuarta. En breve sabremos el número de trastornos que albergará la edición quinta pero no es de esperar que será menos, sino lo contrario. Es manifiesto que la competencia entre terapeutas se hizo y es feroz. Hay cientos y cientos de modelos terapéuticos académicamente identificados, aunque muchos son criticados (8), además de los modelos eclécticos personalizados.

A partir de 1970 y desde una visión médico/biológica de la terapia, los psiquiatras en Occidente establecieron guías clínicas de Terapias Empíricamente Validadas o Respaldadas (EVT: empirically validated treatment; o EST: empirically supported treatment). Las asociaciones de psicólogos se volcaron en la misma tendencia. Pero los fundamentos de las EVT's no son tan sólidos como se pretende. Los estudios comparativos entre tipos de terapias proliferaron pero muy pocos demostraron claras ventajas (9-10), así que se pronunciaron "eficaces" o "vencedoras" a todas, como el "veredicto del pájaro dodo" de Alicia en el País de las Maravillas: "Todos han ganado y todos deben tener premios" $(8,11)$. Desde los años 90 está ganando terreno una visión meta-terapéutica y surgió la pregunta "Si funcionan las terapias, pero no tiene que ver con sus idiosincrasias ¿cuáles son los factores terapéuticos comunes?" La obra de Frank sentó la base de un análisis continuado de los factores comunes en toda la familia de proyectos orientados a producir curación ("healing") de trastornos o problemas psicológicos. Comparó psicoterapia tradicional, terapias de grupo y familiar, tratamiento intraclínico, drogoterapia, medicina, curación religiomágica en sociedades no-industrializadas, cultos y renacimientos. Su conclusión fue que los aspectos compartidos por todas las terapias eficaces son cuatro:

1. Una relación de confianza y con contenido emocional con la persona que quiere ayudar

2. Un entorno "sanador"

3. Un esquema conceptual, razón o mito que ofrece una explicación plausible de los síntomas del paciente y prescribe un ritual o procedimiento para su solución

4. Un ritual o procedimiento que requiere la participación activa tanto del paciente como del terapeuta y que ambos consideran el medio para restaurar la salud. 
ORIGINALES Y REVISIONES

\section{Los Cuatro Grandes}

Michael Lambert (12) elabora sobre el trabajo de Frank y propone, sobre la base de lo que indican los estudios empíricos, que los cuatro factores terapéuticos principales para la mejora de los clientes se identifican como: factores extraterapéuticos, factores comunes, expectación o placebo, y técnicas. Veremos estos factores y su peso relativo en el resultado (varianza) con más detalle:

1. Los factores del cliente ofactores extraterapéuticos $-40 \%$ de la varianza. Son aquellos aspectos del cliente o circunstancias de la vida del cliente que ayudan en la recuperación, independientemente de su participación formal (o estructurada) en la terapia. Son los puntos fuertes del cliente y los elementos de apoyo en su entorno, hasta ocurrencias fortuitas. De hecho, son lo que el cliente aporta a la consulta y lo que influye en su vida fuera de ella. Incluye la persistencia, la confianza, una abuela cariñosa, participar en una comunidad religiosa, el sentido de responsabilidad personal, un nuevo trabajo, suerte en la lotería, una crisis bien llevada... (13). Lambert estimó que los factores del cliente/extraterapéuticos explican un $40 \%$ de la varianza del resultado.

2. Factores de la relación con el terapeuta - $30 \%$ de la varianza.

Coinciden con los que son los "factores comunes" de siempre. Representan un amplio abanico de variables mediados por la relación que se encuentran en terapias de todas las escuelas. Dar importancia, empatía, calor, aceptación, afirmación mutua, animar a tomar riesgos - sólo son unos ejemplos. Se examinaron especialmente las variables aportadas por el terapeuta, las "condiciones esenciales" popularizadas por el humanista Carl Rogers, y se validaron dos de las tres (14).

3. Placebo, esperanza y expectación - $15 \%$ de la varianza.

Lambert encontró que, en parte, la influencia de estos factores deriva del conocimiento que tiene el cliente de que se está tratando, y de una valoración (subconsciente) de la credibilidad de la terapia y sus técnicas. La "expectación" equivale a la condición "d" de Frank, que se refiere a que tanto cliente como terapeuta deben creer en el poder restaurador de los procedimientos o rituales de la terapia (cualquiera).

4. Factores de modelo o técnica $-15 \%$ de la varianza.

Según Lambert, este grupo tiene un peso relativamente modesto en el resultado de la terapia. En un sentido reducido, puede considerarse que estos factores representan las convicciones y procedimientos únicos de un tratamiento específico, por ejemplo la "cuestión milagro" de la terapia breve orientada a soluciones, el uso del genograma en la terapia familiar orientada a Bowen, la hipnosis, la desensibilización sistemática, biofeedback, interpretaciones de transferencia, y sus respectivas premisas teóricas. En un sentido más amplio, abarcan todos los rituales terapéuticos o curativos. Ofrecen una fundamentación organizativa, una explicación teórica 
ORIGINALES Y REVISIONES

de las dificultades del cliente, y establecen estrategias o procedimientos para su resolución. La mayoría prepara al cliente para que tome algún tipo de acción con que ayudarse a sí mismo, y un cambio significativo: desarrollar nuevos entendimientos, sentir emociones diferentes, enfrentarse a temores o cambiar viejos patrones de comportamiento (15).

El fundamento empírico del resultado terapéutico, tanto cuantitativo como cualitativo.

\section{Los datos cuantitativos en general}

Aunque se reconoce que la psicología y, por ende, la psicoterapia debe fundamentarse en hechos y datos clínicamente corroborados, algunos autores de renombre consideran arrogancias científicas y/o teóricas a las pretensiones de los investigadores para "validar" empíricamente la psicoterapia (16). Es cierto que más de seis décadas de evidencia empírica apoyan la eficacia de alguna versión de psicoterapia (17). Las revisiones más relevantes incluyen estudios controlados con miles de pacientes, cientos de terapeutas, un amplio espectro de problemática y muy diversas orientaciones terapéuticas. La terapia es eficaz en el sentido de que, en general, los pacientes tratados evolucionan mejor que los no tratados. Unos 50 meta-análisis desde 1980 indican un promedio de diferencia de un 80\% (1:25) entre tratados y no tratados. Además, el efecto es relativamente rápido: entre ocho y diez sesiones, el 50\% muestra un cambio clínicamente significativo, y una "recuperación" total se produce en un promedio de 25 sesiones, equivalente a seis meses de terapia semanal (18). Los efectos son duraderos (19) especialmente si el cliente los atribuye a sus propios esfuerzos (20), un dato importante que comentaremos en más profundidad en adelante. Pero los meta-análisis comprenden muchos estudios con diseños defectuosos. Sigue en pie - parcialmente - la crítica anterior reflejada de Hans Eysenck: a los 8 meses de tratamiento no se encontraron diferencias entre la terapia psicoanalítica, la terapia cognitiva, y una lista de espera con tratamiento mínimo (17). Si añadimos los estudios realizados en la última década, las conclusiones no varían (21), aunque con excepciones, por ejemplo el meta-análisis pequeño con evaluación personalizada que encontró alguna ventaja de la CBT sobre la terapia psicodinámica (22).

Un dato predominante es que en la mayoría de los estudios comparativos no se encontraron diferencias significativas entre terapias (23). Pero hay que interpretar esta "eficacia compartida" aparente. En primer lugar, diferentes terapias pueden llegar a resultados comparables por vías diferentes. En segundo lugar, se pueden producir diferencias que los diseños de estos estudios no detectaron. En tercer lugar, 
ORIGINALES Y REVISIONES

terapias nominalmente diferentes comprenden factores curativos comunes aunque no enfatizados por la teoría o el cambio, objeto de la escuela correspondiente (24).

\section{Los datos cuantitativos por factores}

Los factores comunes han demostrado ser relevantes para todo tipo de terapia (25). Además de los detalles comentados anteriormente, los siguientes datos concretos ayudan a valorar mejor el rol del terapeuta.

a) Las variables del cliente y los eventos extraterapéuticos (peso 40\%)

Los factores más identificados en los estudios:

- La severidad del trastorno, incluyendo el número de síntomas físicos y psicológicos; estar o no en crisis

- la motivación

- la capacidad para vincularse

- la fuerza del ego y la organización mental

- la medida en que puede pensar de forma psicológica

- la habilidad para identificar un problema focal (26)

- la naturaleza del problema (trastornos de personalidad, esquizofrenia)

- la historia personal (abuso en la infancia, desconfianza personal hacia el profesional)

- la madurez

- la habilidad para involucrarse productivamente en la terapia

Una serie de estudios de Strupp (27) demostró que el terapeuta tiene influencia en todos los casos, aunque en un grado muy determinado por los factores del cliente (para una más completa revisión de la investigación de los factores del cliente véase Imel \& Wampold (28). En general, las variables del cliente no son constantes en el tiempo y pueden cambiar rápidamente, en especial los estilos personales.

La mejora espontánea en casos no tratados o con tratamiento mínimo se produce en una media de $43 \%$ de los casos. Afectan - y por tanto debe considerarse factores terapéuticos - : la duración del trastorno, la presencia de un trastorno subyacente de personalidad, la naturaleza, la fuerza y la calidad del apoyo social, especialmente la relación de pareja y el cambio afectivo, y el tipo de grupo diagnóstico (se observa una mayor remisión espontánea en depresión, seguida por ansiedad, fobias, trastorno obsesivo-compulsivo e hipocondría) (29).

b) Factores de la relación terapéutica (peso 30\%)

La alianza terapéutica (como tal identificada por Freud) es una parte de la relación terapéutica. Al principio la investigación acerca de su carácter e importancia se centró en las condiciones críticas según la tradición (humanista) centrada en el cliente, es decir, la empatía correcta, la estima positiva, el afecto no posesivo, la congruencia de sinceridad, y un mínimo de ataque o culpa. La actividad terapéutica debe dirigirse a estos factores. La relación terapéutica se mide con escalas como la 
ORIGINALES Y REVISIONES

Vanderbilt Therapeutic Alliance Scale (VTAS) (30) que, en el contexto de la psicoterapia dinámica, contempla tres componentes principales de la alianza terapéutica, que son 1) tareas (conductas y procesos dentro de la sesión terapéutica que constituyen el trabajo real de la terapia),2) lazos o vínculos (incluyen las relaciones interpersonales positivas entre terapeuta y cliente, que muestran confianza y aceptación mutua) y 3) objetivos (que deben ser reconocidos y acordados por ambos). (31)

Se ha visto que, en general, el cliente percibe la calidad de la relación terapéutica de forma más correcta que el terapeuta y su valoración se correlaciona más con el resultado final de la terapia. Especialmente relevante es la empatía percibida (hasta $67 \%$ de la varianza, en un estudio con alcohólicos). Los terapeutas más eficaces muestran más conductas positivas y menos negativas. Positivas son: afecto o cariño, entendimiento y afirmación. Negativas son: desprecio o desconsideración, culpar, ignorar o negar, atacar y rechazar.

c) Esperanza y Efectos Placebo (peso 15\%)

Cuanto más elevado el distrés percibido más posibilidad hay de mejora (32). La esperanza de que la terapia funcione es especialmente importante en sus primeras fases (33).

Los efectos placebo relacionados con la esperanza han demostrado poder ser importantes. Cuando se compara placebo con no tratamiento, el placebo da un resultado un $66 \%$ mejor, y cuando se compara tratamiento con no tratamiento: tratamiento $79 \%$ mejor. Pero se han criticado los diseños de estos estudios (34). El National Institute of Mental Health de EE.UU. publicó un estudio en 1989 que compara la terapia cognitivo-conductual y terapia interpersonal con placebo (con trato clínico) que no mostró diferencias significativas (35). En todo caso, el terapeuta debe saber utilizar la esperanza y el factor placebo con el objetivo de incrementar los efectos del tratamiento.

d) Factores de técnica y de modelo de terapia (peso 15\%)

Ejemplos de efectos técnicos específicos:

- La exposición sistemática parece altamente eficaz en fobias simples, agorafobia y compulsiones, pero no en otros problemas como fobias sociales, trastorno de ansiedad generalizada o una combinación de éstas.

- Los resultados de estudios sobre la supuesta eficacia de la terapia cognitiva en trastornos de pánico son cuestionados por los resultados longitudinales decepcionantes (36).

En general, existe poca evidencia de que alguna técnica tenga superioridad. El cada vez más extendido uso de manuales para cada tipo de terapia promueve una mayor uniformidad en las técnicas y los resultados de un terapeuta. Sin embargo, su utilidad es limitada ya que su uso ha demostrado afectar negativamente a la conducta terapéutica de los profesionales, que tienden a mostrar un menor apoyo, menor optimismo, más autoritarismo y posturas defensivas (37). 


\section{Implicaciones para la práctica y la formación}

Según los datos estadísticos estadounidenses (APA, 2012), la mayoría de clientes necesita unas 12 sesiones de terapia, así que - por exigencias del sistema retributivo - el terapeuta debe organizarse sobre esta base. Entre el 20 y el $30 \%$ necesita más de 25 sesiones. Este grupo puede necesitar intervenciones alternativas o aproximaciones más intensivas y multifacetas. Las cifras españolas oscilan entre medias de 11 y 18 sesiones (38).

Los fracasos suelen deberse a una falta de motivación y hostilidad; una historia de relaciones sociales pobres y una postura de receptor pasivo de un procedimiento médico. Se debe identificar estos aspectos al inicio e intentar adecuar las expectativas y las conductas improductivas. Los resultados son más duraderos si la terapia incluye entrenamiento para que 1) el cliente crea en su capacidad de afrontamiento (coping) y 2) tenga una expectativa realista de posibles recaídas temporales después de la terapia. Los terapeutas necesitan reevaluar con cierta frecuencia sus habilidades en cuanto al manejo de los factores comunes en la terapia y mantener un adecuado nivel de expresión y motivación afectiva. El estrés o burnout reduce estas habilidades notablemente y obliga a que el terapeuta sea consciente de sus circunstancias y condiciones personales, de forma constante (39). Cada semana, el terapeuta debe revisar el progreso con el cliente al comienzo de la sesión para que éste le comunique su estado psicológico (40).

\section{La investigación cualitativa acerca de la terapia eficaz}

El cambio en la orientación terapéutica en el mundo desde una individualización terapéutica extrema hacia un eclecticismo integrador implica una combinación individualizada de los factores considerados aplicables. Por ende, es útil tener presente cuáles son los factores que serían aplicables con mayor probabilidad. Una alternativa al análisis estadístico cuantitativo de investigación es la investigación cualitativa (Qualitative Research (IC)), que consiste en el análisis continuado de los datos clínicos y una evaluación constante de hipótesis, básicamente retrospectiva aunque también prospectiva (41). La investigación cualitativa se orienta a "explorar los significados, las variaciones y las experiencias perceptuales de fenómenos". Este enfoque de investigación utiliza la bondad del diseño de investigación, la selección de estrategias de muestreo, conceptos tradicionales de validez, fiabilidad y generalizabilidad, la compatibilidad con aproximaciones cuantitativas, y el análisis informatizado de datos. La investigación clínica es una forma de IC: el terapeuta vuelve a los datos (acumulados) del caso de forma constante para su comprobación y adaptación a una hipótesis que se va mejorando según el desarro- 
ORIGINALES Y REVISIONES

llo del caso. También se llama "aproximación comparativa continuada". Necesita tener una motivación férrea para continuamente intentar falsear la mejor hipótesis, yuxtaponiendo los datos concretos del caso con los datos y diseños antes indicados.

\section{Aspectos de la comunicación terapeuta-cliente}

Se ha demostrado que es aconsejable consultar con el cliente la terapia a seguir, sus formas y los progresos ya que mejora la relación, incrementa la participación del paciente y reduce la presunción de "experto" que pueda tener el terapeuta. Habría que preguntar al cliente si se están cumpliendo sus expectativas, y recoger sus sugerencias para alcanzar mejoras. Se ha visto que la terapia se refuerza en la medida que se pregunte al cliente que resuma lo que se está haciendo en la terapia, el llamado "debriefing" (42). Por ende, el terapeuta necesita habilidades de comunicación específicas para conseguir que el cliente participe activamente en el proceso y tenga lo que Rennie llamó "reflexividad", que consiste en auto-conciencia y auto-control. Con razón terapéutica, muchos estudios se centran en las técnicas de comunicación, y las valoran según cómo el terapeuta ayuda al cliente a contar su historia. La comunicación exitosa se ve muy influenciada por las "prerrogativas conversacionales" del terapeuta. La participación se incrementa con reformulaciones: resúmenes de lo que ha expresado el cliente, estructurado de forma orientada a cambiar la perspectiva. Además, debe controlar las contribuciones no solicitadas y no queridas con técnicas específicas.

El terapeuta necesita aplicar un manejo directivo moderado de la conversación con que sustituir la dicotomía (repetitiva) del "sí, pero" y el enfrentamiento de posiciones, por el "también, además" de la búsqueda participativa de soluciones compartidas.

Todas las terapias cognitivas e interpersonales o sistemáticas consisten en la reestructuración de ideas maladaptativas que han demostrado producir problemas para el cliente. Sin embargo, el terapeuta debe valorar abiertamente la autenticidad de estas ideas y pensamientos, sin criticarlos o tildarlos de negativos o inútiles, ya que esto significaría culpar al cliente de su trastorno y desanimarle en cuanto a la terapia.

\section{Los Factores Comunes, y aplicar la investigación acerca de su importancia.}

\section{A. El Cliente como factor común decisivo}

Para muchos terapeutas es difícil aceptar el hecho de que los clientes se curan a sí mismos. Sin embargo, la capacidad para curarse a sí mismo es el factor común más potente en psicoterapia (43). Como se comentó antes, la evidencia apunta a 
que todas las terapias consiguen una mejora parecida (44). Sin embargo, que haya una eficacia similar de todas las terapias no significa que el terapeuta pueda hacer lo que le dé la gana. Para que la terapia sea eficaz tanto el terapeuta como el cliente deben estar convencidos de que ésta puede ser eficaz, tomar en serio sus propios roles, las técnicas y los modelos que están aplicando (45). Aunque la experiencia del terapeuta no parece ser un factor decisivo (46-47), las cualidades personales que se expresan en el trato y se reflejan en la calidad de la relación y la activación de los recursos del cliente sí lo son. En total, los factores de éste son responsables del $70 \%$ de la varianza del resultado, calculado de la forma siguiente: placebo $(15 \%)+$ la mitad del factor de la relación $(15 \%)+$ sus factores propios $(40 \%)(11,48)$. Por ende, los "magos" con poderes especiales curativos son los clientes. Los terapeutas organizamos el escenario y servimos de asistentes que aportan las condiciones bajo las cuales la magia puede funcionar. Ayudamos a movilizar, canalizar y enfocar la magia del cliente.

Como decía, puede que cueste reconocer que las diferencias en el éxito terapéutico entre terapeutas con mucha experiencia y los que empiezan son pequeñas. Los libros de auto-ayuda, la terapia informatizada y por internet, o unas clases terapéuticas de grupo pueden ser igualmente efectivos en determinados trastornos que la ayuda profesional, por ejemplo en la depresión (49), lo cual enfatiza el poder auto-curativo del cliente. Se han demostrado eficaces o beneficiosos:

- La auto-expresión y la auto-revelación del cliente (50)

- Hablar en una grabadora es igual de efectivo que la terapia cognitiva en cuanto a la resolución de experiencias traumáticas (51)

- La escucha empática sólo es algo menos eficaz que una intervención psicoterapéutica (en pacientes antes de una cirugía por cáncer de mama) (52)

Estos resultados indican que el cliente tiene una capacidad considerable para generar su propio cambio, una vez encontrado el método estructurado adecuado para ello. La maduración, o sea, el paso del tiempo puede resolver de por sí muchos trastornos, como el trastorno de personalidad límite, la conducta asocial y agresiva, el alcoholismo o la adicción al tabaco. Prochaska formuló sobre este fenómeno su teoría de los estadios de cambio (Stage theory of Change; en 1:227 y ss.) ¿Puede que todo cambio sea auto-cambio, y la terapia sencillamente sea un auto-cambio con tutor?

Se calcula que la recuperación espontánea llega a un 40\% (16) y en general es facilitada por el contacto con personas que no son profesionales de la salud mental. Parece que los que están en terapia necesitan personas ajenas para hablar y aclarar el tema, en mayor medida que los que no están en terapia.

El 60\% de los clientes que piden la primera consulta informa de una mejora desde que hacían la cita y antes de acudir por primera vez. (53). Muchos mejoran con la primera consulta, lo cual demuestra que la causa real de la mejora (al menos este tipo de mejora) no es la terapia sino el esfuerzo global de cambio por parte del cliente. 
ORIGINALES Y REVISIONES

\section{Algo más sobre los placebos}

No sólo son importantes los efectos placebo en cuanto a la esperanza (ver antes). La agencia personal, es decir, tomar parte activa en el asunto, se despierta por técnicas específicas. Cuanto más activo y específico el procedimiento, más creíble resulta para el cliente y más movilizará su esperanza, energía, creatividad y potencial de auto-curación intrínseca. Por tanto: los rituales ayudan, pero ¿qué rituales? Aquí también la fuerza del efecto placebo se ha demostrado en muchos estudios, tanto en medicina como en psicoterapia, por ejemplo el estudio NIMH de depresión, antes referido (54).

Es preciso diferenciar clases de placebo (55). En medicina se considera que el placebo activo es aquella aplicación que produce cambios fisiológicos y sensaciones corporales, sin producir curación efectiva. (Greenberg, en (1). Por tanto, las técnicas de relajación no ayudan al cliente con el cambio, mientras las técnicas que evocan emociones fuertes pueden servir de ayuda (fumar rápido, la exposición en fobias).

\section{La relación terapéutica}

Según la explicación tradicional, esta relación puede tener efecto terapéutico por sí mismo. No está claro el mecanismo, pero en parte puede que tenga efecto por:

- Ser una "experiencia emocional correctiva", así que tendría efecto curativo inherente por corregir contactos o experiencias tóxicas del pasado

- Crear un ambiente que refuerza conductas más apropiadas sin perder identidad propia o someterse a otros

- Crear nuevas oportunidades de aprendizaje

- Movilizar la estructuración psíquica propia y cómo se concibe a sí mismo el cliente

Sin embargo, no hay evidencia directa para considerar a estos posibles efectos como una explicación suficiente para el cambio terapéutico provocado. En la actualidad, se considera que la calidad de la relación es sólo un recurso más que soporta o enfoca los esfuerzos del cliente para curarse. Aporta un ambiente seguro para este fin. La relación es creada e interpretada por el cliente, subjetivamente, por ello que tanto su implicación como la(s) resistencia(s) son parámetros importantes. No debe subestimarse la implicación activa del cliente, incluso cuando parezca pasivo u ofreciendo resistencia. Sí que hay evidencia de que el éxito terapéutico se correlaciona con la valoración de la relación por parte del cliente, más que la del terapeuta. Por tanto, no es el conocimiento o la habilidad del profesional lo que 
determina el éxito. El cliente percibe la empatía - parte importante de la alianza terapéutica - de forma diferencial: no es un concepto global e unidimensional y en consecuencia el terapeuta necesita orientarse sobre lo que le importa al cliente. Tipos de empatía: cognitiva, afectiva, de compartir, o nutritiva.

\section{La participación del cliente.}

Decía que la voluntad de involucrarse de la persona es, junto con la alianza, la mayor variable del resultado terapéutico (70\%). Para ella, la motivación es importante, pero sólo aquella que se ve desde la perspectiva del cliente. Por ende, clarificar y consensuar los objetivos de la terapia se asocia con un resultado positivo. La esperanza de mejora se relaciona con la motivación, pero la evidencia indica que tiene importancia sólo en el inicio de la terapia (56).

\section{La opinión del cliente}

Los propios clientes consideraron que son de más ayuda (57):

Las características facilitadoras del terapeuta, poder descargar el distrés, la autocomprensión y sentirse animado a empezar una práctica gradual.

En depresión y después de 8 sesiones de CBT o terapia psicodinámica/interpersonal en depresión, los clientes valoraron más (58): la conciencia incrementada, aprender soluciones de problemas, sentirse reconfortado, y el contacto personal. En el estudio clásico de Sloane, Staples, Cristol, Yorkston \& Whipple (59) que comparó psicoanálisis con terapia conductual, los clientes consideraron más decisivos para el buen resultado cómo encajaba la personalidad del terapeuta y cómo les llevaba gradualmente a un entendimiento de sí mismo. En general, los esfuerzos activos no específicos, propios del cliente, se han demostrado esenciales para solucionar los problemas.

\section{Reflexividad y agencia del cliente}

El término reflexividad, acuñado por el psicólogo humanista David Rennie, de la Universidad de York en Canadá en los años 1990-2000, refiere a la autoconsciencia y la agencia dentro de la misma, por ende un valor introspectivo que también refleja en qué medida el cliente está dispuesto a trabajarlo. Se observó 
ORIGINALES Y REVISIONES

que los clientes suelen ser activos durante las sesiones pero a un nivel encubierto, e intentan conducir al terapeuta en la dirección que ellos quieren. Aunque los terapeutas cometan errores, los clientes los ignoran y hacen su selección para utilizar lo que les parece beneficioso. Se debe estimular el pensamiento generador del cliente. Es preciso que se experimente el cambio en la percepción de las cosas no sólo cognitivamente sino con todo el cuerpo, todo el ser: el cambio "sentido" (60). Si sólo fuese cambio intelectual, la gente no necesitaría a los terapeutas: se le explicaría lo que tendría que hacer, sin más. Pero vienen a terapia los que no consiguen poner en práctica lo sabido.

Las vías hacia este cambio sentido incluyen (61):

- La extinción (por exposición)

- La práctica conductual y repetitiva

- Actividades experimentales de búsqueda a través de tareas en casa

- Las intervenciones paradójicas

- Comparar las experiencias actuales con las cogniciones disfuncionales

- Revisar las conexiones entre las experiencias de la infancia y las actuales

- Poner en palabras o articular la experiencia particular

\section{Técnicas terapéuticas específicas}

En vista del demostrado encaje con las necesidades y posibilidades de determinados grupos de clientes, la aplicación de técnicas específicas tiene ventajas. El terapeuta debe preguntarse: ¿Por qué este cliente busca terapia? Las razones son varias:

1. La persona está estresada o queda enganchada en ciclos de pensamiento rumiante e improductivo (62).

2. Bajo estrés, las personas suelen usar conocimientos viejos para resolver problemas nuevos, lo cual limita la creatividad que se necesitaría para encontrar soluciones eficaces (50)

3. Cuando el problema es resistente y ya han fracasado varios intentos de solución, las personas tienden a exhibir el coping disfuncional de individuos impotentes o incapaces (63), perseverando o bien probando otras soluciones pero de forma aleatoria, errática o desesperada.

4. Faltan recursos en el entorno (64)

5. No pueden tomar la distancia y perspectiva necesaria

6. No tienen con quien hablar y quien verdaderamente les escuche

7. Les faltan las habilidades personales mínimas 


\section{Implicaciones para la práctica de la terapia}

Considerando lo anterior, el terapeuta debe esforzarse por escuchar más al cliente, respetar más su marco de referencia, y buscar una colaboración genuina con él (que es más que sólo conseguir la participación y el cumplimiento). Equivocadamente, muchas terapias buscan el modelo médico, donde el terapeuta es el experto que "diagnostica" la naturaleza del problema ("trastorno") y seguidamente "prescribe" el "tratamiento". Los EVT's se basan en este modelo, con sus manuales que no dejan espacio a una elección genuina por parte del cliente y perfilan la colaboración con éste según la definición que da la terapia, además de unos esquemas para el desarrollo de la misma sobre las que siempre decide sólo el terapeuta.

\section{B. La relación terapéutica}

El concepto de terapia de Carl Rogers se distinguía del de Freud, por afirmar que no es un encuentro entre un experto (terapeuta) y un acólito (cliente), sino un encuentro existencial que activaría la sanación innata y el potencial de crecimiento innato a cada persona. Pero Rogers se limitaba a contemplar la contribución del terapeuta. Strong reducía la relación terapéutica a la influencia social. Según él, sería crucial lo que el cliente opina acerca del terapeuta. (65) Desde el conductismo, Skinner (66) consideraba al éxito terapéutico como el resultado de un proceso biológico de aprendizaje, por ende las técnicas son decisivas. Hans Eysenck (6) apretaba aún más: Sólo funcionan las terapias conductuales, no valen las "charlas": las terapias conversacionales. A pesar de esto, la "charlaterapia" sigue siendo el método preferido por algunos terapeutas, por comodidad, indisciplina o falta de conocimiento de métodos más estructurados.

Hace bastantes décadas, Gelso y Carter (67) hacían una advertencia aún válida: la terapia no es sólo una relación de trabajo, de llevarse bien mutuamente, sino necesita ser real e incluir compromiso mutuo. Con el desarrollo de diversas escalas para medir la alianza terapéutica empezó la investigación empírica de su calidad y cantidad en contextos diferentes, así como de su correlación con el resultado terapéutico.

Actualmente, se consideran componentes esenciales de la alianza:

1. Los vínculos o lazos interpersonales entre los participantes, incluidos el terapeuta

2. El acuerdo alcanzado acerca de los fines de la terapia

3. La colaboración en tareas terapéuticas (33)

Las investigaciones descubrieron que la alianza tiene, además, otros componentes, entre ellos el apoyo percibido, la auto-observación, las contribuciones negativas por ambas partes y la asociación colaboradora percibida. No existe consenso acerca de su definición ni del contenido necesario de cada uno de estos 
ORIGINALES Y REVISIONES

componentes, pero sí acerca de su importancia para todas las terapias, incluso en farmacoterapia con contacto de apoyo mínimo (17).

La alianza temprana (las sesiones tres a cinco) es predictor significativo del resultado final del tratamiento. En terapia individual, más que en grupo, la alianza fluctúa. Con experiencia y entrenamiento, el terapeuta podrá adecuarse al cliente (68).

La empatía se correlaciona con la efectividad del terapeuta (69) y con el éxito (70). Las diferentes intervenciones tienen una asociación mixta con el resultado (71). También es importante el momento: aunque pueden tener eficacia en otros momentos, tenían efecto negativo sobre la relación inicial el apoyo, la exploración de pensamientos y emociones, y la búsqueda de información (72), posiblemente porque aún no se había establecido la alianza y por tanto el cliente mantuvo su "burbuja defensiva de intimidad". El terapeuta debe dosificar las interpretaciones porque pueden provocar una reacción defensiva del cliente por sentirse criticado (73).

Además de las características generales de la mayoría de las relaciones terapéuticas, el terapeuta necesita adecuar al cliente varios factores (74):

- el nivel de formalidad (casual vs. profesional; afectiva vs. cognitiva)

- la auto-revelación (aunque puede ser contra-producente)

- el calor y la empatía demostrados

- el apoyo (directividad y confrontación)

- la focalización de tópicos (problemas sintomáticos vs. conflictivos)

- el nivel de focalización (conductual versus descubridor)

- el ritmo del trabajo terapéutico

- cómo hablar de material intra-terapia o extra-terapia

En el cliente evasivo no habría que insistir mucho en que debe describir y mostrar emociones o que exprese afecto porque provocaría distancia y frialdad. En este caso se le refuerza para que él seleccione los tópicos a tratar pero se abstiene de respuestas interpretativas o desafiantes, mientras el terapeuta actúa con interés pero sin muestras explícitas de apoyo ni de amistad (75). El cliente dado a la oposición se beneficiaría más de una relación terapéutica donde percibe al terapeuta como un socio o compañero al mismo nivel y que ejerce poco control directo, acepta las selecciones oposicionales hechas, y desarrolla tareas creadas y decididas conjuntamente (76).

\section{Las contribuciones del cliente: colaboración e implicación}

La implicación personal y la motivación se asocian con mejoras terapéuticas (77) así como la apertura (poder asimilar lo que ofrece el terapeuta) y la auto- 
exploración y sentir afecto (implicación). La implicación del cliente es mejor predictor del éxito que las actitudes o técnicas del terapeuta (30). Por tanto, son más cruciales para la alianza las comunicaciones que muestran una alta implicación del cliente que las del terapeuta, y los clientes con mejores alianzas producen más comunicaciones (78).

La calidad de la relación terapéutica se merma por reacciones defensivas o evasivas, la hostilidad o una personalidad hostil-dominante. Clientes con un estilo provocador o evocador pueden resultar difíciles, y primero debe tratarse la hostilidad (79). No todas las verbalizaciones negativas indican una mala relación terapéutica, y es posible utilizarlas para mejorarla, reconociendo los logros del cliente, por ejemplo el insight que demuestra y la comprensión que tiene del proceso de la terapia (80).

\section{Conclusión}

La psicoterapia no es un arte al azar, sino un conjunto de factores identificables y valorables en cuanto a su influencia sobre el resultado final. Muchos de los datos y hechos arriba reflejados son intuitivos y fáciles de incorporar en la práctica, pero otros muchos no lo son tanto. Por ende, el profesional de la psicoterapia se ve obligado a adaptar su quehacer y, sobre todo, su auto-evaluación a la realidad demostrada del cliente, y los factores relevantes del caso. Su primera obligación es aplicar la terapia más eficaz posible. Forma parte de las obligaciones terapéuticas la auto-actualización constante, lo que implica flexibilizar las ideas rígidas y deshumanizadas que algunas veces se imponen con el argumento falaz de una mayor eficacia de las técnicas terapéuticas uniformizadas. El terapeuta debe ser, ante todo, respetuoso con su más valioso material: los factores del cliente.

El terapeuta no nace con un don con que analizar y solucionar los problemas psicológicos de los demás. Se tiene que hacer a si mismo, se lo tiene que trabajar mucho y seguir trabajándolo durante toda su vida profesional. Es cierto que necesita, desde el principio, la motivación de querer implicarse en el bienestar psíquico de las personas, pero aunque esta motivación tal vez no sea tan común, no es suficiente sino un mero prerrequisito.

BIBLIOGRAFÍA:

(1) Hubble MA, Duncan BL, Miller SD. The Heart and Soul of Change: What works in Therapy. Washington DC, APA; 1999. doi:10.1037/11132-000 
ORIGINALES Y REVISIONES

(2) Tyrer P, Silk KR (Eds.). Cambridge Textbook of Effective Treatments in Psychiatry. Cambridge: Cambridge University Press; 2008. Pp.106 y.ss. doi:10.1017/CBO9780511544392

(3) American Psychological Association . Resolution on the Recognition of Psychotherapy Effectiveness, Approved August 2012. http://www.apa.org/news/press/releases/2012/08/resolutionpsychotherapy.aspx

(4) Rozenzweig S. Some implicit common factors in diverse methods in psychotherapy. Journal of Orthopsychiatry1936; 6: 412-15. doi:10.1111/j.1939-0025.1936.tb05248.x

(5) Bergin AE, Lambert MJ. The evaluation of therapeutic outcomes. In Garfield SL \& Bergin AE (Eds.) Handbook of psychotherapy and behavior change, $2^{\text {nd }}$ ed. New York: Wiley;1978

(6) Eysenck HJ. The effects of psychotherapy: An evaluation. Journal of Consulting Psychology 1952;16:319-24. doi:10.1037//0022-006X.60.5.659

(7) Frank JD. Persuasion and Healing: A Comparative Study of Psychotherapy, 1961, 1973, 1991. Baltimore: Johns Hopkins; 1991

(8) Siev J, Huppert J, Chambless DL. The dodo bird, treatment technique, and disseminating empirically supported treatments. The Behavior Therapist 2009; 32, 69-75.

(9) Elkins DN. Empirically supported treatments: The deconstruction of a myth. Journal of Humanistic Psychology, 2007;47(4), 474-500

(10) Norcross JC, Newman CF. Psychotherapy integration: Setting the context. In Norcross JC \& Goldfried MR (Eds.) Handbook of psychotherapy integration pp.3-45. New York: Basic Books; 1992

(11) Luborsky L, Singer B, Luborsky L. Comparative studies of psychotherapies: Is it true that "Everybody has won and all must have prizes?" Archives of General Psychiatry 1975;32: 9951008. doi:10.1001/archpsyc.1975.01760260059004

(12) Lambert MJ. Implications of outcome research for psychotherapy integration. En Norcross JC y Goldfried MC (Eds.). Handbook of psychotherapy integration (pp.94-129). New York: Basic Books; 1992

(13) Duncan BL, Hubble MA, Miller SD. Stepping off the throne. The Family Therapy Networker 1997; 21(3):22-31,33

(14) Kirschenbaum H, Jourdan A. The current status of Carl Rogers and the PersonCentered Approach. Psychotherapy: Theory, Research, Practice, Training, 2005;42(1):37-51. doi:10.1037/0033-3204.42.1.37

(15) Miller SD, Duncan BL, Hubble MA. Escape from Babel: Toward a unifying language for psychotherapy practice. New York: Norton; 1997

(16) Lambert MJ. Manual-based treatment and clinical practice: Hangman of life or promising development? Clinical Psychology: Science and Practice, 1998;5:391-5. doi:10.1111/j.1468-2850.1998.tb00160.x

(17) Lambert MJ, Bergin AE. The effectiveness of psychotherapy. En Bergin AE y Garfield SL (Eds.) Handbook of psychotherapy and behavior change, $4^{\text {th }}$ ed., pp.143-189. New York: Wiley; 1994

(18) Smith MI, Glass GV, Miller TI. The benefits of psychotherapy. Baltimore: Johns Hopkins University Press; 1980

(19) Kadera SW, Lambert MJ, Andrews AA. How much therapy is really enough? J. Of Psychotherapy: Practice and Research 1996;5:10

(20) Nicholson RA, Berman JS. Is follow-up necessary in evaluating psychotherapy? Psychological Bulletin 1983; 93:261-78. doi:10.1037//0033-2909.93.2.261 
(21) Hunot V, Churchill R, Silva de Lima M,Teixeira V. Psychological therapies for generalised anxiety disorder. Cochrane Database Syst Rev. 2007 Jan 24;(1):CD001848. doi:10.1002/14651858. CD001848.pub3

(22) Ougrin D. Efficacy of exposure versus cognitive therapy in anxiety disorders: systematic review and meta-analysis. BMC Psychiatry 2011;11:200. doi:10.1186/1471-244X-11-200

(23) Staines GL. The relative efficacy of psychotherapy: Reassessing the methods-based paradigm. Review of General Psychology 2008;12(4):330-43. doi:10.1037/1089-2680.12.4.330

(24) Spielmans GI, Pasek LF, McFall JP. What are the active ingredients in cognitive and behavioral psychotherapy for anxious and depressed children? A meta-analytic review. Clinical Psychology Review,2007; 27(5): 642-54. doi:10.1521/jscp.1994.13.2.189

(25) Hoffart A, Borge FM, Sexton H, Clark DM. The role of common factors in residential cognitive and interpersonal therapy for social phobia: A process-outcome study. Psychotherapy Research 2009; 19(1):54-67. doi:10.1080/10503300802369343

(26) Tolin DF. Is cognitive-behavioral therapy more effective than other therapies? A metaanalytic review. Clin Psychol Rev. 2010;6:710-20. Epub 2010 May 25. doi:10.1016/j.cpr.2010.05.003

(27) Strupp HH. Success and failure in time-limited psychotherapy. Arch of General Psychiatry 1980;37: 363-79, 595-603, 708-16, 831-41, 947-54. doi:10.1001/archpsyc.1980.01780180109014; doi:10.1001/archpsyc.1980.01780190106013; doi:10.1001/archpsyc.1980.01780210105011

(28) Imel ZE, Wampold BE. The importance of treatment and the science of common factors in psychotherapy. In Brown SD \& Lent RW (Eds.) Handbook of counseling psychology (4th ed.). Hoboken, NJ: Wiley \& Sons; 2008

(29) Garfield SL. Research on client variables in psychotherapy. In Garfield SL \& Bergin AE (Eds.) Handbook of psychotherapy and behavior change, 4th ed. New York: Wiley; 1994

(30) Windholz JJ, Silberschatz G. Vanderbilt psychotherapy process scale: A replication with adult outpatients. Journal of Consulting and Clinical Psychology 1988;56: 56-60. doi:10.1037//0022006X.56.1.56

(31) Schapira K, Roth M, Kerr TA, Gurney C. The prognosis of affective disorders: The differentiation of anxiety states from depressive illnesses. British Journal of Psychiatry 1972;12:17581. doi:10.1192/bjp.121.2.175

(32) Gaston L, Marmar CR, Thompson LW, Gallagher D. Relation of patient pretreatment characteristics to the therapeutic alliance in diverse psychotherapies. Journal of Consulting and Clinical Psychology 1988;56(4):483-9. doi:10.1037//0022-006X.56.4.483

(33) Bordin ES. The generalizability of the psychoanalytic concept of the working alliance. Psychotherapy: Theory, Research and Practice,1979;16:252-60. doi: 10.1037/h0085885

(34) Frank JD, Gliedman LH, Imber SD, Stone AR, Nash EH. Patients' expectancies and relearning as factors determining improvement in psychotherapy. American Journal of Psychiatry 1959;115:961-8

(35) Wampold BE, Bhati KS. Attending to the omissions: A historical examination of the evidenced-based practice movement. Professional Psychology: Research and Practice 2004;35:56370. doi:10.1037/0735-7028.35.6.563

(36) Elkin I, Shea MT, Watkins JT, Imber SD, Sotsky SM, Collins JF, et al. NIMH Treatment of Depression Collaborative Research Program: General effectiveness of treatments. Archives of General Psychiatry 1989;46:971-83. doi:10.1001/archpsyc.1989.01810110013002 
ORIGINALES Y REVISIONES

(37) Milrod B, Busch F. Long-term outcome of panic disorder treatment: A review of the literature. Journal of Nervous and Mental Disease 1996;184:723-30. doi:10.1097/00005053-19961200000002

(38) Echeburúa E, Salaberría K, de Corral P, Polo-López R. Terapias psicológicas basadas en la evidencia: limitaciones y retos de futuro. Revista argentina de clínica psicológica 2010; XIX: 247-56

(39) Campagne DM. When therapists run out of steam: professional boredom or burnout? Revista de Psicopatología y Psicología Clínica - Spanish Journal of Clinical Psychology RPPC 2012;17(1):75-85

(40) Henry WP, Strupp HH, Butler SF, Schacht TE, Binder JL. Effects of training in time-limited dynamic psychotherapy: Changes in therapist behavior. Journal of Consulting and Clinical Psychology 1993;61:434-40. doi:10.1037//0022-006X.61.3.441

(41) Montero I, León OG. Sistema de clasificación del método en los informes de investigación en Psicología. International Journal of Clinical and Health Psychology 2005;5(1):115-27

(42) Rennie D. Qualitative analysis of the client's experience of psychotherapy: The unfolding of reflexivity. In Tourmanian S \& Rennie D (Eds.) Psychotherapy process research: Pragmatic and narrative approaches (pp.211-33) Newbury Park, CA: Sage; 1992

(43) Todd TA, Joanning H, Enders L, Mutchler L, Thomas F. Using ethnographic interviews to create a more cooperative client-therapist relationship. Journal of Family Psychotherapy 1990;1(3):51-63. doi:10.1300/j085V01N03_04

(44) Bohart AC, Talman K. How clients make therapy work: The process of active selfhealing. Washington, DC: American Psychological Association; 1999. doi: 10.1037/10323-000

(45) Wampold BE. Methodological problems in identifiying efficacious psychotherapies. Psychotherapy Research 1997;7:21-44. doi:10.1080/10503309712331331853

(46) Norcross JC. Eclectic psychotherapy: an introduction and overview. In Norcross JC (Ed.) Handbook of eclectic psychotherapy (pp 3-24) New York: Brunner/Mazel; 1986

(47) Svartberg M, Stiles TC. Comparative effects of short-term psychodynamic psychotherapy: a meta-analysis. Journal of Consulting and Clinical Psychology 1991;59:704-14. doi:10.1037//0022-006X.59.5.704

(48) ChristensenA, Jacobson NS. Who (or what) can do psychotherapy: The status and challenge of nonprofessional therapies. Psychological Science 1994;5:8-14. doi:10.1111/j.1467-9280.1994. tb00606.x

(49) Stein DM, Lambert MJ. Graduate training in psychotherapy: Are therapy outcomes enhanced? Journal of Consulting and Clinical Psychology 1995;63:182-96. doi:10.1037//0022006X.63.2.182

(50) Pennebaker JW. Emotion, disclosure, and health: An overview. In JW PenneBAKER (ED.) Eмотіол, disclosure, and health (pp.3-10). Washington DC:American Psychological Association; 1995. doi:10.1037/10182-015

(51) Pennebaker JW. Opening up: The healing power of confiding in others. New York: Morrow; 1990

(52) Segal DL, Murray EJ.Emotional processing in cognitive therapy and vocal expression of feeling. Journal of Social and Clinical Psychology 1994;13:189-206. doi:10.1521/jscp.1994.13.2.189

(53) Burton MV, Parker RW, Wollner JM. The psychotherapeutic power of a "chat": A verbal response modes study of a placebo attention control with breast cancer patients. Psychotherapy Research 1991;1:39-61 
(54) Lawson D. Identifying pretreatment change. Journal of Counseling and Development 1994;72:244-8. doi:10.1002/j.1556-6676.1994.tb00929.x

(55) Campagne DM. La gradación del efecto placebo; un estudio $\mathrm{N}=1$ doble ciego, con belladona homeopática 30C. Psiquis2002; 23(4):20-6. ISSN 0210-8348

(56) Horvath AO. The therapeutic relationship: From transference to alliance. In Session 1995;1:7-17. doi:10.1002/(SICI)1097-4679(200002)56:2<163::AID-JCLP3>3.3.CO;2-4

(57) Perotti LP, Hopewell CA. Expectancy effects in psychotherapy and systematic desensitization: A review. JSAS: Catalog of Selected Documents in Psychology 1980;10 (Ms.No.2052)

(58) Elliott R, James E. Varieties of client experience in psychotherapy: An analysis of the literature. Clinical Psychology Review1989; 9:443-67. doi:10.1016/0272-7358(89)90003-2

(59) Sloane RB, Staples FR, Cristol AH, Yorkston NJ, Whipple K. Short-term analytically oriented psychotherapy vs.behavior therapy. Cambridge MA: Harvard University Press; 1975

(60) Grawe K. Research-informed psychotherapy. Psychotherapy Research 1997;7:1-20. do i: $10.1080 / 10503309712331331843$

(61) Gendlin ET. The experiential response. In Hammer E (Ed.) Use of interpretation in treatment (pp 208-27) New York: Grune \& Stratton; 1968

(62) Gendlin ET. Focusing-oriented psychotherapy. New York: Guilford Press; 1996

(63) Ward TB, Finke RA, Smith SM. Creativity and the mind. New York: Plenum Press; 1995

(64) Bandura A. (1997). Self-efficacy. New York: W.H.Freeman;1997

(65) Heppner P, Rosenberg J, Hedgespeth J.Three methods in measuring the therapeutic process: Clients' and counselors' constructions of the therapeutic process versus actual therapeutic events. Journal of Counseling Psychology 1992;39:20-31. doi:10.1037//0022-0167.39.1.20

(66) Skinner BF. Recent issues in the analysis of behavior. Englewood, NJ: Prentice-Hall; 1985

(67) Gelso CJ, Carter JA. Components of the psychotherapy relationship: Their interaction and unfolding during treatment. Journal of Counseling Psychology 1994;41:296-306. doi:10.1037//0022-0167.41.3.296

(68) Mallinckrodt B, Nelson ML. Counselor training level and the formation of the psychotherapeutic working alliance. Journal of Counseling Psychology 1991;38:133-8. doi:10.1037//00220167.38.2.133

(69) Lafferty P, Beutler LE, Crago M. Differences between more and less effective psychotherapists: A study of select therapist variables. Journal of Consulting and Clinical Psychology 1989;57:76-80. doi:10.1037//0022-006X.57.1.76

(70) Bachelor A. Comparison and relationship to outcome of diverse dimensions of the helping alliance as seen by client and therapist. Psychotherapy: Theory, Research and Practice 1991;28:534-49. doi: 10.1037/0033-3204.28.4.534

(71) Beutler LE, Machado PPM, Allstetter Neufeldt SA. Therapist variables. In BA \& SL Garfield (Eds.) Handbook of psychotherapy and behavior change (pp: 229-69) New York: Wiley; 1994

(72) Kivlighan DM. Relation between counselors' use of intentions and clients' perception of working alliance. Journal of Counseling Psychology 1990;37:27-32. doi:10.1037//0022-0167.37.1.27

(73) Piper WE, Azim FA, Joyce SA, McCallum M. Transference interpretations, therapeutic alliance and outcome in short-term individual psychotherapy. Archives of General Psychiatry 1991;48:946-53. doi:10.1001/archpsyc.1991.01810340078010 
ORIGINALES Y REVISIONES

(74) Lazarus AA. Tailoring the therapeutic relationship, or being an authentic chameleon. Psychotherapy: Theory, Research, and Practice 1993;30:404-7. doi:10.1037/0033-3204.30.3.404

(75) Dolan R, Arnkoff D, Glass C. Client attachment style and the psychotherapist's interpersonal stance. Psychotherapy: Theory, Research, and Practice 1993;30:408-12. doi:10.1037/00333204.30.3.408

(76) Beutler L, Consoli A. Matching the therapist's interpersonal stance to clients' characteristics: Contributions from systematic eclectic psychotherapy. Psychotherapy: Theory, Research, and Practice 1993;30:417-22. doi: 10.1037/0033-3204.30.3.417

(77) Orlinsky DE, Grawe K, Parks BK. Process and outcome in psychotherapy - noch einmal. En Garfield BA \& Garfield SL (Eds.) Handbook of psychotherapy and behavior change (pp 270-376). New York: Wiley; 1994

(78) Reandeau SG, Wampold BE. Relationship of power and involvement working alliance: A multiple-case sequential analysis of brief therapy. Journal of Counseling Psychology 1991;38:10714. doi:10.1037//0022-0167.38.2.107

(79) Gaston L.The concept of the alliance and its role in psychotherapy: Theoretical and empirical considerations. Psychotherapy 1990;27:143-53. doi:10.1037/0033-3204.27.2.143

(80) Adler G. The psychotherapy of core borderline psychopathology. American Journal of Psychotherapy 1993;47:193-205 